\title{
Conferencias de Therapeutica Clinica
}

\section{Dr. Ulysses Paranhos}

(Devemos á gentileza do Dr. Ulysses Paranhos a publicação, nesta Revista, das Conferencias de $T e^{-}$ rapseutica Clinica que o ilustre professor livre na Universidaide do Rio de Idneiro vem realizandc, aos domingos, com raro brilho, na 2.a Medicina de Mulheres da Sta. Casa de Misericordia.

$A$ conferencia que se vae ler é a primeira do seu curso, que a Revista de Medicina pretende publicar integralmente.)

Senhores:

As arterites chronicas tiveram, nos ultimos annos, fóros de realeza em clinica. Com os estudos de Traube e Von Basch, na Allemanha, Pctain e Huchard, èm França e Riva-Rocci, na Italia, o phantasma da hypertensão, o principal sindrome desta entidade morbida, arıdou na móda e todo o velho e todo o epicurista gabou-se de ser ou vir a ser um doente de suas arterias.

Presentemente, com os trabalhos de Pal, de Vienna e da escola franceza representada, sobretudo, por Vaquez e seus discipulos, a questão das arterites foi collocada nos devidos termos e a sua therapeutica fundamentada em dados mais scientificos e positivos.

$\mathrm{O}$ que se chama uma arterite crolnica?

A arterite cronica é uma lesão degenerativa dos vasos arteriaes, cujo processo anatomo pathologico differe conforme a degenerescencia se localiza para o lado dos grandes ou dos pequenos vasos. 
Se os grandies vasos são atacados, particularmente a aorta, regis ta-se uma degenerescencia das tunicas da arteria e mota-se a formação de placas a principio gelatiniformes e ,mais tarde, duras, esclerosas, calcareas; são os chamados atheromas, sendo denominada de atheromasia a lesão caracterizada pelo presença de atheromas. A arterite cronica, das arterias medias, e pequenas, se dejigna sob o nome de arterio escleróse e possue como substractum uma transfcirmação fibrosa ccrm espessamento da tunica muscular do vaso.

As arterites cronicas dependem de uma toxi-infecção lenta, actuando constantemente e, por longo tempo, sobre as paredes vasculares e oriundas de venenos fabricados pelo proprio organismo ou, então, de ir fecções microbianas ou intoxicações accidentaes.

No numero das auto intoxicações figuram as differentes affecções clascificadas no termo confuso de artritismo. Nas intoxicações exogenas enfileiram-se, em primeira plano, o alcool, o tabaco e o chumbo.

No numero das infecções capazes de provocar a arterite cronica, mostra-se em primeiro plano a lues, que segundo as recentes estatisticas de Vaquez é a responsaval pela maicria dos casos dessa affecção.

Emfim a ve'hice, assigna'ando o declltio das funcções vitaes, determinando a miopragia do organismo inteiro é suscertive de causar arterites cronicas e isso vemos, particularmente, demois do cincoentenario, nos estafados physica e moralmente, nos que viveram sem moderação e nos portadores de uma debilidade orgalnica dos vasos. Nestes, as arterias enferrujam-se precocemente, repetindo a phrase pitoresca de Peter.

As arterias atacadas por um processo inflamatorio cronico, quando são accessiveis ao exame, apresentam-se sem elasticidadre, dura, com dilatações e nodosidades.

Quando a arterite cronica interessa as arterias do cerebrc, notam-se a presença d evertigens, zumbidos de ouvido, cefalća, perturbações visuaes e sensitivo-motoras; qualrdo affecta os vacos dos membros abdominaes o doente queixa-se ce dôr, formigamento, podendo-se apresentar o phenomeno da claudicação intermitente.

A atheromasia aortica revela-se por um certo numero de symptomas funccionaes e signaes physicos, que precisam ser conhecidos.

Existem dôres retro-esternaes, violentas, com irradiações vara o pescoço e braço, dores essas seguidas de surtos dyspneicos reflexos, com ponto de partida aortico e exercendo sua acção sobre o vago. Notam-se ainda vertigerns, barulho ncs ouvidos, e phenomenos subsidiarios do systema neuro glandular da vida organica. 
No ponto de vista da propedeutica physica se observará que a aorta se mostra dilatada, a crossa ultrapassaindo o esterno, as pulsações augenentadias e perceptiveis á palpação e a escuta accusa a percepção de um clangôr á segunda bulha.

$\mathrm{Na}$ phase avançada da arterite cronica estabelece-se a cachexia arterial, ou em outros termos, estabelece-se um estado de depauperamento geral e progressivo, que leva o coração a grande insufficiencia, com terminação pela morte.

De um modo geral pode-se sintetizar o revolver da arterite cromica no seguinte conceito de Huchard: "— começa por uma intoxicação, man" tem-se por uma intoxicação e termina-se por uma intoxicação".

\section{EXPOSIÇÃO DE UIMA OBSERVAÇÃO CLINICA DE ARTERITE GRONICA (1)}

Mulher de 50 annos, preta, casadia, brasileira, domestica.

Antecedentes hereditarios: - sem importancia.

Antecedentes pessoais: - relata ter gozado bôa saúde. Nega contagio venereo-sifilitico. Não tem filhos vivos. Perdeu 4 entre abortos $e$ nati-mortos e outros $4 \mathrm{em}$ diferentes idades' e de causa ignorada. Diz ter tido reumatismo articular agudo. Amnesia no que se refere á noção do tempo.

Historia actual — Ha algum tempo começou sentir-se donete: dôres nas pernas, especialmente esquerda, cefaléa, tonturas. As dores dos membros são acompanhadas de formigamento, sente entorpecimento e retracção das pernas, que se produz quando quer exercer algutma actividade, e que é acompanhada de formigamento. Vê-se obrigada a procurar apoio nestas occasiões, para não cahir. Já apresentou edema dos membros inferiores hoje desaparecidos em virtude de estar deitada. Sensações de zumbido no ouvido esquerdo. Dôres abdominaes extendendo-se á região lombo dorsal esquerda. Constipação rebelde do ventre. Polakiuria.

Exame physico: mulher de côr preta, regularmente constituida no aspecto geral. Pela inspecção, cicatrizes de queimaduras nos pulsos devidas á applicação de rémedios qualrudo tem reumatismos. Deformação bilateral dos membros inferiores, que tomam o aspecto de uma adaga turca. Lipoma na região dorso lombar direita.

(1) Tomada pelo doutorando José Augusto Lefévre. 
Ganglios epitracleanos bastante volumasos do lado direito. Esternalgia e tibialgia acentuadas.

Exame do aparelho circulatorio: - Pulso 80, denunciando pouca elasticidade das arterias.

Arterias humeraes e radiales sinuosas e um tanto resistentes. É perceptivel, ao nivel do umbigo, a aorta cbdominal.

Prressão arterial (methodo Riva-tRocci): - $M x-170 ; M n-160$.

Choque da ponta no $6 .^{\circ}$ espaço intercostal, á esquerda da linha mamilar. Area cardiaca augmentada. Escuta: sôpro sistolico perceptivel no foco mitral; clangor da 2. bulha perceptivel no foco a.ortico.

Aparelh,o respiratorio - Hypersonoridade, em relação com a idade, na base do pulmão direito. Murmurio respiratorio apagado em toda extensão do thorax.

Aparelho abdominal — nada dignno de registo.

Aparelha genito-urinario - urina albuminosa, diminuição dos saes, raros cylindros hyalinos.

O exame radiologico revelou augmento da aorta e outros signaes de aortite cronica; Wassermann negativa.

\section{CONSIDERAÇÕES THERAPEUTICAS}

A observação acima referida pode-se diagriosticar como sendo de uma arterite cronica de origem luetica, manifestando-se sob a forma anatomo-clinica de atheromasia e arterioesclerose.

A Wassermann negativa não invalida a ideia de lues, que tem em seu favor os abortos, a nati-mortalidade da descendencia da doente; a presença de ganglios epitrocleanos e a tibialgia e esternalgia acentuadas.

O diagnostico de arterite cronica fundamenta-se numa serie de signaes e symptomas que, lendo a observação, chamamos a vossa attenção; vertigens, claudicação intermitente, zumbido nos ouvidos, hypertensão, ar terias duras e sinuosas, augmento da area aortica, clangor aortico, etc.

Firmado o diagnostico de arterite cronica, qual o tratamento a se instituir em nossa doente?

Em primeiro lugar é pieciso confessar que contra o processo esceroso, uma vez e'le iniciado, rada podemos fazer, apenas é possivel im- 
pedir que elle evolva e se agrave, ou, então, attenuar as suas consequenvcias maleficas scibre o resto do organismo.

Para isso, devemos seguir as seguintes normas, baseiadas nas noções etiologicas da doença e no seu processo anatomo-clinico de desenvolvimento:

1) - Inistituir um regimen hygienico dietetico racional;

2) - estabelecer uma therapeutica geral das desordens funcionaes:

3) - acudir aos symptomas alarmantes que se apresentarem;

Como regra devida, deve a nossa doente manter-se muma actividade relativa, sendo condemnado o repouso constarite no leito. Pequenos serviços domesticos, que não determinem a fadiga, são recommendaveis.

As refeições serão repetidas e moderadas, afim de se evitar a repleção do estomago, sobretudo ao jantar, que pode ser a causa de reflexos dyspneicos, congestivos e angustiosos. Deve dormir 8 horas sobre 24 .

Nessa classe de doentes convem manter-se activa a curculação peripherica, afim de poupar trabalho ao coração: são, pois, acchuselhaveis os banhos tepidos seguidos de fricção com luva de crina ou baeta embebida em alcool canforado.

E' preciso lembrar que os esclerosos são muito sensiveis ao frio e suas consequencias nelles são a receiar-se, attendendo á grande miopragia das suas visceras.

Nestas circumstancias, é preciso agazalha-los sufficientemente, evitando as correntes de ar e a passagem brusca de um ambiente quente para outro frio, que pode determinar, particularmente, affecções pulmonares graves.

Ventre livre, meio seguro de diminuir as auto intoxicações. O melhor medicamento, neste caso é o oleo de ricino tomado uma a duas capsulas todas as noites ao deitar-se. Os salinos não conveem, pela ibercia intestinal que se segue á- súa administração. Purgativos drasticos são perigosos; casos de morte têm sido registrados com a sua preconização, consoante observações do Dr. Antonio Pedro e outros. O fumo, devido á acção toxica que a inicotina exerce sobre os sistemas nervoso e circulatorio, será condemnado. Chá e café, parcimoniosamente, bem fraco, duas a tres vezes ao dia.

Nada de athmosphera viciadia; ar livre, puro, bem oxygenado.

Da maior importancia é o regimen alimentar; erros alimentares são, em geral, os causadores dos accidentes graves registados em doentes como a nossa e, não raros são os reponsaveis pela morte prematura nós casos de esclerose vascular 
Casos existem que possuem quasi o cunho experimental.

Senhora esclerosa, com insufficiencia cardio-renal. $O$ seu medico, um dos nossos mais reputados clinicos, aconselha um regimem hypo-toxico, racional. Adoente revolta-se, acha-se fraca, não fica bôa. Novo medico lé chamado. Este muda a dieta: os accidentes são foreiros, diz elle, de uma nutricção insufficiente. Almoce bife, ovos, vínho Bordéos. Decorridas 48 horas a infeliz fallece de coma uremico!

Senhora edosa, cachexia arterial grave. Prescrevemos regimem de leite e fructas. Desobedece e, num domingo, rebella-se e come feijão e creme dre óvos. $\mathrm{Na}$ terça-feira repousava da vida, após uma uremia sem remedio.

Os factos relatados dão a media do quanto vale, no caso que nos occupa, uma dieta racional.

Faltando a lesão renal, deve-se prohibir as carnes, as gorduras e os alimentos ricos em cal aos arterio esclerosos ie os atheromatosos simples?

Huchard tinha horror á carme; ha nisso um pouco de exagero. Em dorentes com rim são pode-se deixar usar carne fresca, de açougưe, só na refeição da manhã. Qualnto ás gorduras e aos alimentos ricos em cal, podem ser ingeridos sem receio. Vaquez e Laubry affirmam e com razão que elles não exercem influencia perniciosa sobre os doentes. Abandonem-se os alimentos ricos em purinas e em acido oxalico.

Nos doentes com accidentes de nefrite cronica, como a nossa, convem. prescrever uma alimentação de accordo com a determinação renal; hypoclorurado na nefrite hydropigenica; hipoazotado na azotemia, como é o nosso caso, consoante provam as pesquizas de laboratorio.

No nosso caso é recommemdavel um regimem constituido por baitatas, arroz cozido, legumes verdes, fructas e pão torrado na quantidade de 100 a 200 grs. por dia. Leite, 200 a 400 quotidianamente, figurando como alimento accesorio.

Delineado o regimem, quaes são os medicamentos a prescrevermos contra o processo geral anatomo-clinico da arterite cronica?

Dedntre elles o principal é o iodo, que a despeito de ter contra sí certo numero de contradictores, mais theoricos que praticos, guarda ainda em clinica sua realeza.

Actua pela sua acção vaso dilatadora, activando a circulação dos tecidos e a diurése, e facilitando, dest’arte, a eliminação doz residuos toxicos.

Age, tambem, pela sua acção anti-toxica geral, atteruando os effeitos das toxinas sobre os elementos celulares, frenando a tendenca esclerogenica do processo inflamatorio arterial. 
Deve-se receitar de preferencia os preparados iodados de absorpção lenta, aquelles de influencia constante e tolerancia perfeita.

Por via gastrica é recommendavel a lipoiodina, tres comprimidos por dia; ou a saiodina, inas mesmas doses. São succezaneos organicos dos iodetos de efficacia e tolerancia perfeitas, não ćando lugar ao àparecimento de phenomenos de iodismo, embora se os utilize prolcngadamente. Por via hypodermica prefiram-se sempre os oleos iodados: o lipoiodol, a iodipina, que se injectartá na dose media de 2 a 5 cc. por dia. Aconselhaveis por se applicar bôa porção do metalloide em pequena quantidade de vehiculo. $\mathrm{E}$ 'esse o methodo que vae ser submettida a nossa diente.

Convem preconizarmos os alcalinos conjunctamente com a medicação iodada. Delles, o mais indicado é o citrato de sodio: urolithico, desacidificante e anti-coagulante, prevenindo as tromboses. Pode-se usar um papel de 2 grs. ean um copo de agua pela manhã e á noite.

No "que se refere á D’Arsonvalisação e'é diathermia, methodos muito gabados pelos especialistas; os seus resultados restão longe das esperanças despertadias nos primeiros ensaios. Contra taes processos, șe levantaram duas autoridades maximas em physiotherapia: Bordier e André Broca. No que se refere aos banhos carbogasozos, reservas grandes devem tambem ser feitas à respeito dos interessados cantarem lôas aos seus bellos successos.

Trendo-se certeza ou supposição de que o processo de aortite é subsidiario da sifilis, colsvem prescrever, como therapeutica geral, o tratamento anti-luetico?

Quando a infecção é relativamente recente, sim; quando artiga, qualquer medicação, neste sentido, é improficua e ás vezes, mal tolerada.

A medicação melhor é peló bi-iodeto de mecrurio, serie de 20 injecções cada mez, durante tres mezes cada anmo. Preferir a via intra-muscular.

Ahi fica, em largos traços, a therapeutica geral das arterites cronicas, cabendo-nos agora, entrar na sua cura symptomatica.

O symptoma maximo, o que mais preoccupa ao clinico, no decurso das arterites cronicas, é a hypertensão.

Ha valntagem em combater sempre a hypertensão?

Conforme. Quando ella não é accusada senão pelo manometro, quando não traz nenhum desequilibrio na vida do doente, é antes uma reacção de defeza, e, portando, merece ser respeitada.

Quando, entretanto se revela por vertigem, zumbidos no ouvido, accessos anginoides, crices vasculares e outros sintomas assaz conhecidos. merece therapeutica adequada. 
Uim medicamento a ser applicado, nesta opportunidade, pelo seu reffeito real sobre o apparelho vascular, é a papaverina, que, com entusiasmo, $\mathrm{Pal}$ (cle Vienna), considera entre os mais efficazes hypotensores.

Pode-se applicar a seguinte formula:

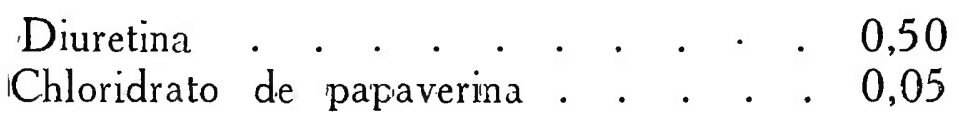

F. Em uma capsula, $\mathbf{n} \cdot{ }^{\circ} 30$, tome 3 ao dia.

Não se proporcionará a papaveriná nas lesões resclerósas dos rins, sendo no entanto, indicado todas as vezes que a hypertensão fôr de origem funcional esparmodica (hypertensão transitoria e formas moderadas da hypertensão arterial).

É ainda aconselhavel, nestes casos, o silicato de sodio, que provoca uma queda da pressão arterial e o desapparecimento das vertigens, cefaléa e albuminuria.

É recommendavel a seguinte formula:

Solução xaroposa de silicato de sodio. 15 grs. Agua distillada de fl. de laranjeiras 250 grs.

F. Uma colher de sộpa, em meio copo de agua, tres vezes por dia, durarite um a dois mezes; suspender quinze dias e recomeçar de novo.

Loeper elogia, como um excellente hypotensor, a tintura de alho. Não possumos experiencia pessoal sobre o assumpto. Tomam-se XX gotas por dia em um calice de agua.

Nas crises da hypertensão intensas, em que se precisa intervir rạidamente, é dé bom aviso lançar mão de outros hypotensores, cujo effeito se manifesta pouco tempo após a sua preconização. É o caso da doente que nos occupa.

Eintre esses figura o nitrito de amylo e a trinitrina.

O nitrito de amylo, o ether amylo-nitroso, é o medicamento de preferncia nas hypertensões: diffunde-se rapidamente no sangue e provoca uma vaso-dilatação intensa acompanhada de queda da pressão sanguinea, consequencia desta vaso-dilatação sanguinea.

Usa-se em inhalações, sobretudo, nos surtos anginoides; não se utilizará desse corpo nos arterios escleroses congestivos.

A trinitrina, ether trinitino da glycerina, emprega-ze exclusivamente em solução alccolica a 1/100. É Lum vaso-dilatador energico, seirdo o homem 
muito sensivel á sua influencia. Poucos momentos após o seu emprego, observa-se uma hyperhemia intensa e baixa da pressão respectiva. A acção da trinitrina é sempone passageira, o habito se manifesta em pouco tempo.

Usa-se em injecções hypodermicas sob a seguinte formula :

Sul. centesimal de trinitrina . . . . IV gotas

Agua distillada esterilizada. . . 2 cc.

En uma empôlla. Para injecção sub-cutanea.

Internamente adopta-se a formula abaixo que prescrevemos para o nosso caso:

Solução alcoolica centesimal de trinitrina XL gotas Agua distilada. . . . . . . . 200 grs.

Duas a quatro colheres das de sobremesa por dia, continuar dez dias, interromper dez dias e recomeçar dez dias.

É necessario não esquecer, repretimos, que estes hypotensivos são de acção rapida, mas de effeito tranusitorio. Pode-se considera-los medicamentos de occasião. Para combater a hypertensão. de um modo definitivo temos: o regimem, os iodicos e papaverina.

Havendo lesão renal presta, como hypotensor reaes serviços, a theobromina na dose de 1 gr. por dia, á qual se ajuntará igual dose de phosphato neutro de sodio, para augmentar a tolerancia para o remedio.

Renon, Irsessa forma de hypertensor, aconselha, e com vantagem a tintura de cratego, que se pode utilizar por muito tempo e que possue um poder sedatido sobre o systema sympathico, provocando, quando administrada prolongadamente uma real vaso-dilatação.

Na cachexia arterial de Huchard, derradeiro acto do grande drama de fallencia organica que occasiona a arterite cronica e em que as toxinas que envenenam a economia não são mais produzidas pela decompocicãa dos ralimentos, porém fabriciadas por ella proprio e em que os residuos toxicos que envenenam o sangue não podem ser mais removidos nem pelos iodicos, nem pelos vaso-dilatadores, nem pelos diureticos, faz-se mister recorrer a medidas energicas se quizermos salvar o organismo que morre aos pedaços: nesta situação veem á bailla a sangria, os purgativos, o sôro glycosado, as injecçõecs de oxygeno, que aliviam e prolongam uma existencia de sofrimento, que, só a morte, com o seu repouso final, poderá dar um supremo alivio. 


\section{Considerações em torno de quatro casos de Esthiomene}

\section{(Ulcus Rodens - Ulcus vulvae chronicum)}

No decurso destes ultimos mezes nada menos de quatro casos de Esthiomene, clinicamente diagnosticados e confirmados pelos exames histo pathologicos, foram registados no Serviço do Professor N. Moraes Barros .

Gynecopathia rara, a incidencia destes quatro casos, que mereceram de nossa parte especial attenção, estava a exigir a sua divulgação, pois especialistas existem que perlustram pela actividade clinica sem opportunidade da constatação de um só caso.

Sobretudo nos preoccupou a quəstão etiologica, porisso que meticulosamente investigamos as duas principaes causas incriminadas - a tuberculose e a syphilis. Emquanto que alguns, como Bracht e schade empr sstam ao bacillo tuberculoso um papel preponderante, outros como Küstner veem na syphilis o principal responsavel, e finalmente outros, como Weinbrenner, admittem uma tuberculosa desenvolvida em base syphilitica.

Ha ainda quem vislumbre na blennorrhagia um elemento etiologico de rəlevancia, como Stein e Heinemann.

A raridade da affecção em cottejo com a extrema disseminação da gonorrhéa autorisa_nos excluil-a como agente etiologico. Ao demais nos nossos quatro casos, dois delles se manifestaram em senhoras honestas e não contaminadas. As duas restantes embora apresentassem a infeccão neisseriana não eram prostitutas, de sorte quə pelo menos entre nós, não se justifica a expressão de Labhardt de que o ulcus rodens culmina de frequencia entre as prostituidas. E, de facto, a infeccão gonococcica é aviltada pela quasi totalidade dos autores.

A hypothese que de principio nos pareceu mais sympathica foi da origem tuberculosa da ulceração. Assim, tivemos o cuidado de realisar esfregaços com a secreção das ulceras e submettel-os á exames, realjsados que foram pelo Dr. L. Salles Gomes, e todos negativos. Por outro

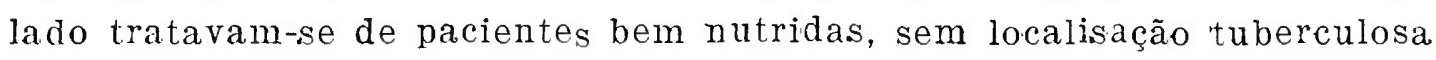
em qualquer orgão, de molde que excluimos a possibilidade de uma manifestạão secundaria. 